NONCOMMUTATIVE GEOMETRY

AND QUANTUM GROUPS

BANACH CENTER PUBLICATIONS, VOLUME 61

INSTITUTE OF MATHEMATICS

POLISH ACADEMY OF SCIENCES

WARSZAWA 2003

\title{
DIFFERENTIAL CALCULI OVER QUANTUM GROUPS
}

\author{
G. J. MURPHY \\ Department of Mathematics, National University of Ireland, Cork, Ireland \\ E-mail: g.j.murphy@ucc.ie
}

\begin{abstract}
An exposition is given of recent work of the author and others on the differential calculi that occur in the setting of compact quantum groups. The principal topics covered are twisted graded traces, an extension of Connes' cyclic cohomology, invariant linear functionals on covariant calculi and the Hodge, Dirac and Laplace operators in this setting. Some new results extending the classical de Rham theorem and Poincaré duality are also discussed.
\end{abstract}

1. Introduction. This paper concerns the differential calculi that arise in the setting of compact quantum groups. As soon as these calculi were discovered by S. L. Woronowicz [11] he and others recognized that they do not fit into the framework of noncommutative geometry developed by A. Connes [1]. In fact, something entirely new arises in the quantum group setting, as we shall see below. It is already clear that a deep and significant extension of Connes' theory will be required to deal with these new calculi.

The subject of this paper is joint work of the author and J. Kustermans and L. Tuset, see $[6,7]$. The reader should consult these works for further details of results or concepts discussed here, in the case that no explicit reference is given.

The paper is an expanded version of a talk delivered at the conference Noncommutative Geometry and Quantum Groups held at the Banach Center in Warsaw in September 2001. The author would like to express here his thanks to the organizers for their kind invitation to speak at the conference.

The paper begins with a brief section that defines basic terms and explains in what sense differential calculi over quantum groups differ not only from the calculi that appear in the classical theory of manifolds, but also from the calculi appearing in the theory of noncommutative geometry of Alain Connes. The following section, Section 3, introduces the concept of a twisted graded trace. This is a sort of "volume integral" on a differential calculus and extends Connes' concept of a graded trace. The latter concept is no longer adequate to deal with the "integrals" that occur in the quantum

2000 Mathematics Subject Classification: 46L05, 46L65, 16W30, 28 B32.

The paper is in final form and no version of it will be published elsewhere. 
group setting. Twisted graded traces are a sort of analogue of a KMS state, whereas a graded trace is an analogue of a tracial state. We discuss in this section an extension of Connes' theory of cyclic cohomology that bears a similar relationship to the differential calculi and integrals that occur in the quantum group framework as ordinary cyclic cohomology does to the differential calculi and integrals considered by Connes in his version of noncommutative geometry. In Section 4 we discuss left-invariant linear functionals on left-covariant differential calculi. Covariant calculi are the most important in the theory and invariant functionals are natural objects of study in their setting. We discuss conditions that ensure that a left-invariant linear functional is a twisted graded trace. Our results state that, in a sense, the objects one would naturally consider to be the "volume integrals" in this theory are indeed twisted graded traces. In Section 5 we consider the Laplace and Dirac operators. We also discuss a Hodge-type decomposition and show how this implies a version of the classical de Rham theorem identifying the cohomology spaces of the de Rham complex with corresponding spaces of harmonic forms. In the penultimate section, Section 6, we introduce some conditions that ensure the existence of a Hodge operator and that enable us to prove a version of Poincaré duality. The final section, Section 7 , is concerned with the question of obtaining a $K$ cycle, in the sense of Connes, from the differential calculi that occur in the quantum group framework. Our results here again point to the need for an extension of existing theories.

2. Differential calculi. This brief section is concerned with basic notations and terminology and discusses in outline how the theory of differential calculi over quantum groups is different from the classical theory of forms on a manifold and from the theory of forms and integrals occurring in Connes' version of noncommutative geometry.

Let $\mathcal{A}$ be a unital algebra (all algebras and linear spaces are over the complex field $\mathbf{C}$ ). A pair $(\Omega, d)$ is a differential calculus over $\mathcal{A}$ if the following conditions hold:

1. $\Omega=\oplus_{n=0}^{\infty} \Omega_{k}$ is a positively-graded algebra such that $\Omega_{0}=\mathcal{A}$;

2. $d: \Omega \rightarrow \Omega$ is a graded derivation of degree one such that $d^{2}=0$;

3. For each positive integer $n, \Omega_{n}$ is the linear span of the elements of the form $a_{0} d a_{1} \cdots d a_{n}$ and $d a_{1} \cdots d a_{n}$, where $a_{0}, \ldots, a_{n} \in \mathcal{A}$.

Of course, this concept extends the usual idea of the calculus of differential forms on a manifold, where the underlying algebra $\mathcal{A}$ is the algebra of smooth complex-valued functions on the manifold.

There is always at least one differential calculus over an arbitrary unital algebra, namely, the universal differential calculus. Although this plays an important role in our results (see [6] for details), we shall have no need to consider it in this paper. At this point we should observe that a problem with differential calculi in the general setting of arbitrary algebras is their non-uniqueness. There may be many calculi over the algebra being considered that seem to be equally nice or suitable (or equally problematical!). This is the case for the Hopf *-algebra underlying the quantum group $S U_{q}(2)$. In this case, there are two 4-dimensional bicovariant differential calculi. (Covariant calculi are discussed in Section 4.) 
If $\omega_{k}$ and $\omega_{l}$ are a $k$-form and an $l$-form on a differential manifold, then we have near commutativity in the sense that

$$
\omega_{k} \wedge \omega_{l}=(-1)^{k l} \omega_{l} \wedge \omega_{k}
$$

where $\wedge$ denotes the usual exterior product of forms. Nothing like this is true in the quantum setting. For instance, in the first 3-dimensional calculus that Woronowicz constructed over the quantum group $S U_{q}(2)$ (see [11]), we have 1-forms $\omega(0), \omega(1), \omega(2)$ for which

$$
\omega(1) \wedge \omega(0)=-q^{4} \omega(0) \wedge \omega(1)
$$

and

$$
\omega(2) \wedge \omega(0)=-q^{2} \omega(0) \wedge \omega(2) .
$$

Here the scalar $q$ is a certain deformation parameter lying in the punctured interval $[-1,+1] \backslash\{0\}$. In fact, not even this kind of twisted commutation relation holds between arbitrary $k$ - and $l$-forms $\omega_{k}$ and $\omega_{l}$ in a differential calculus in the general quantum group setting.

In Connes' theory of noncommutative geometry it is also the case that Equation (1) no longer holds; that is, we do not have the graded commutativity that holds in manifold theory. However, equality under the "integral sign" does hold: that is, we have

$$
\int \omega_{k} \wedge \omega_{l}=(-1)^{k l} \int \omega_{l} \wedge \omega_{k},
$$

where $\omega_{k}$ and $\omega_{l}$ are $k$ - and $l$-forms in a differential calculus over an algebra and $\int$ is an "integral". More precisely a linear functional $\int$ satisfying Equation (2) is called a graded trace. Graded traces are absolutely central in Connes' theory - they play the role there of "volume integrals".

However, in the quantum group setting the "volume integrals" that arise are not graded traces, as first observed by Woronowicz in [11]. In fact, as we showed in [6], they are more like "graded KMS functionals" in a sense that we make precise in the next section, where we discuss the properties of these functionals.

\section{Twisted graded traces and an extension of Connes' theory of cyclic co-} homology. Let $(\Omega, d)$ be a differential calculus over a unital algebra $\mathcal{A}$. We say that a linear functional $\int: \Omega \rightarrow \mathbf{C}$ is a twisted graded trace if there exists an algebra automorphism $\sigma: \Omega \rightarrow \Omega$ of degree zero such $\sigma d=d \sigma$ and

$$
\int \omega_{k} \wedge \omega_{l}=(-1)^{k l} \int \sigma\left(\omega_{l}\right) \wedge \omega_{k},
$$

for all forms $\omega_{k} \in \Omega_{k}$ and $\omega_{l} \in \Omega_{l}$. As before, we are using $\wedge$ to denote the multiplication operation on $\Omega$. We call $\sigma$ a twist automorphism for $\int$.

When we discuss covariant calculi in the next section we shall see that the volume integrals that occur in that setting (the quantum group setting) are twisted graded traces in this sense. For example, the volume integral defined by Woronowicz on his first 3dimensional left-covariant calculus over $S U_{q}(2)$ is a twisted graded trace. The same is true for the $4 D_{+}$bicovariant calculus over $S U_{q}(2)$. 
If $\tau$ is a KMS state (at inverse temperature $\beta=1$ ) on a $\mathrm{C}^{*}$-algebra $A$, then there is a dense *-subalgebra $\mathcal{A}$ and an algebra automorphism $\sigma: \mathcal{A} \rightarrow \mathcal{A}$ such that $\tau(a b)=$ $\tau(\sigma(b) a)$, for all $a, b \in \mathcal{A}$. It is this aspect of the KMS condition that our twisted graded traces generalize. However, the analogy is closer even than this. We refer the reader to Section 7 of [7], where it is shown that, in certain circumstances, an automorphism group can be associated to a twisted graded trace that plays a role relative to it similar to the role played by a modular automorphism group relative to a corresponding KMS state.

One of Connes' important ideas is to show a correspondence between cyclic co-cycles and triples $\left(\Omega, d, \int\right)$ consisting of differential calculi $(\Omega, d)$ and certain graded traces $\int$ on $\Omega$. The graded traces in this correspondence need to be closed in the sense that $\int d=0$ and to be $N$-dimensional, for some non-negative integer $N$, in the sense that $\int \omega_{k}=0$, for all $\omega_{k} \in \Omega_{k}$, where $k \neq N$. If this is the case, then the $N+1$-linear functional $\varphi: \mathcal{A}^{N+1} \rightarrow \mathbf{C}$ defined by setting

$$
\varphi\left(a_{0}, a_{1}, \ldots, a_{N}\right)=\int a_{0} d a_{1} \cdots d a_{N}
$$

is a cyclic co-cycle. Moreover, every $N$-dimensional cyclic co-cycle $\varphi$ arises from a triple $\left(\Omega, d, \int\right)$ in this fashion.

If $\int$ is a twisted graded trace on a differential calculus $(\Omega, d)$, assumed to be closed and $N$-dimensional, and $\varphi$ is defined as in Equation (3), then $\varphi$ will not be, in general, a cyclic co-cycle. However, it turns out that it will be a cycle in another cohomology theory, as we showed in [6]. The new cohomology theory generalizes Connes' cyclic cohomology and has many of the features, including all of the important basic ones, of Connes' theory. We discuss now how this new theory is defined.

First, let's recall the outlines of Connes' cyclic cohomology for a unital algebra $\mathcal{A}$. Begin by denoting by $C^{n}$ the linear space of all $n+1$-linear functionals on $\mathcal{A}$. Denote by $b: C^{n} \rightarrow C^{n+1}$ the usual Hochschild co-boundary operator defined, for $\varphi \in C^{n}$, by the equation

$$
\begin{gathered}
(b \varphi)\left(a_{0}, \ldots, a_{n+1}\right)=\sum_{i=0}^{n}(-1)^{i} \varphi\left(a_{0}, \ldots, a_{i-1}, a_{i} a_{i+1}, a_{i+2}, \ldots, a_{n+1}\right) \\
+(-1)^{n+1} \varphi\left(a_{n+1} a_{0}, a_{1}, \ldots, a_{n}\right) .
\end{gathered}
$$

This defines a complex $\left(C^{n}, b\right)_{n}$ whose associated cohomology is the Hochschild cohomology $H H^{*}(\mathcal{A})$ of $\mathcal{A}$. To obtain cyclic cohomology we need to introduce the permutation operator $\lambda: C^{n} \rightarrow C^{n}$ defined, for $\varphi \in C^{n}$, by setting

$$
(\lambda \varphi)\left(a_{0}, \ldots, a_{n}\right)=(-1)^{n} \varphi\left(a_{n}, a_{0}, \ldots, a_{n-1}\right) .
$$

Denote by $C_{\lambda}^{n}$ the subspace of $C^{n}$ of all elements $\varphi$ for which $\lambda \varphi=\varphi$. Then $\oplus_{n} C_{\lambda}^{n}$ is invariant under $b$ and therefore we get a new complex $\left(C_{\lambda}^{n}, b\right)_{n}$ by restriction of $b$. The cohomology associated to this complex is the cyclic cohomology $H_{\lambda}^{*}(\mathcal{A})$ of $\mathcal{A}$.

Suppose now $\sigma: \mathcal{A} \rightarrow \mathcal{A}$ is an algebra automorphism of $\mathcal{A}$ and we shall associate a cohomology to the pair $(\mathcal{A}, \sigma)$. First, we replace the Hochschild co-boundary operator by 
another operator $b_{\sigma}: C^{n} \rightarrow C^{n+1}$ defined by modifying Equation (4) as follows:

$$
\begin{gathered}
\left(b_{\sigma} \varphi\right)\left(a_{0}, \ldots, a_{n+1}\right)=\sum_{i=0}^{n}(-1)^{i} \varphi\left(a_{0}, \ldots, a_{i-1}, a_{i} a_{i+1}, a_{i+2}, \ldots, a_{n+1}\right) \\
+(-1)^{n+1} \varphi\left(\sigma\left(a_{n+1}\right) a_{0}, a_{1}, \ldots, a_{n}\right) .
\end{gathered}
$$

A direct calculation shows that $b_{\sigma}^{2}=0$. We also need a modified version $\lambda_{\sigma}: C^{n} \rightarrow C^{n}$ of the permutation operator, defined by changing Equation (5) as follows:

$$
\left(\lambda_{\sigma} \varphi\right)\left(a_{0}, \ldots, a_{n}\right)=(-1)^{n} \varphi\left(\sigma\left(a_{n}\right), a_{0}, \ldots, a_{n-1}\right) .
$$

Now denote by $C_{\lambda}^{n}(\mathcal{A}, \sigma)$ the linear subspace of $C^{n}$ consisting of all elements $\varphi$ for which $\lambda_{\sigma} \varphi=\varphi$. The space $\oplus_{n} C_{\lambda}^{n}(\mathcal{A}, \sigma)$ is invariant under $b_{\sigma}$. Using this, we get a complex $\left(C_{\lambda}^{n}(\mathcal{A}, \sigma), b_{\sigma}\right)_{n}$ by restriction of $b_{\sigma}$. The cohomology of this complex is our new twisted cyclic cohomology of the pair $(\mathcal{A}, \sigma)$ and is denoted by $H_{\lambda}^{*}(\mathcal{A}, \sigma)$.

Of course, when $\sigma=\iota$, the identity automorphism of $\mathcal{A}$, our new cohomology theory reduces to Connes' theory: $H_{\lambda}^{*}(\mathcal{A}, \iota)=H_{\lambda}^{*}(\mathcal{A})$.

Let us briefly explain the intuitive difference between our more general theory and Connes' theory. If $\varphi \in C_{\lambda}^{0}(\mathcal{A})$, then $\varphi$ is a co-cycle - that is, $b \varphi=0$-if and only if, $\varphi\left(a_{0} a_{1}\right)=\varphi\left(a_{1} a_{0}\right)$, for all $a_{0}, a_{1} \in \mathcal{A}$; in other words, $\varphi$ is a trace. One then thinks of the co-cycles in $C_{\lambda}^{n}(\mathcal{A})$ as generalized traces. (Terminology in this connection is somewhat ambiguous in the literature, since the use of the term trace often includes an assumption of positivity of the functional involved. Of course, we make no such assumption here.)

Now suppose that $\varphi \in C_{\lambda}^{0}(\mathcal{A}, \sigma)$. Then $\varphi$ is a co-cycle; that is, $b_{\sigma} \varphi=0$, if and only if, $\varphi\left(a_{0} a_{1}\right)=\varphi\left(\sigma\left(a_{1}\right) a_{0}\right)$, for all $a_{0}, a_{1} \in \mathcal{A}$. Thus, one can think of co-cycles in $C_{\lambda}^{n}(\mathcal{A}, \sigma)$ as some kind of generalized KMS functionals.

It is natural to ask whether there is a version of Hochschild cohomology for the pair $(\mathcal{A}, \sigma)$ and it turns out that there is. Indeed, this is important for our general theory. If one defines $C^{n}(\mathcal{A}, \sigma)$ to be the linear subspace of $C^{n}$ consisting of all elements $\varphi$ for which $\lambda_{\sigma}^{n+1} \varphi=\varphi$, then $\oplus_{n} C^{n}(\mathcal{A}, \sigma)$ is invariant for $b_{\sigma}$ and we get an induced complex $\left(C^{n}(\mathcal{A}, \sigma), b_{\sigma}\right)_{n}$ whose corresponding cohomology $H H^{*}(\mathcal{A}, \sigma)$ is our twisted Hochschild cohomology of the pair $(\mathcal{A}, \sigma)$. As with the cyclic theory, if $\sigma=\iota$, we reduce to the usual theory: $H H^{*}(\mathcal{A}, \iota)=H H^{*}(\mathcal{A})$.

Remarkably, the general features and properties of Connes' cyclic cohomology theory extend to the general setting of the pair $(\mathcal{A}, \sigma)$. For example, one gets $S, B$ and $I$ operators and a long exact sequence relating them as in Connes' theory. One can show these facts by re-tracing the steps in Connes' original proofs and suitably modifying them. A more efficient approach is possible, however, using the cyclic category $\Lambda$.

Suppose now $(\Omega, d)$ is a differential calculus over the unital algebra $\mathcal{A}$ and that $\int: \Omega \rightarrow \mathbf{C}$ is an $N$-dimensional, closed, twisted graded trace. Define the $N+1$-linear functional $\varphi$ on $\mathcal{A}$ by the formula in Equation (3). Then $\varphi \in C_{\lambda}^{N}(\mathcal{A}, \sigma)$, where $\sigma$ is the restriction to $\mathcal{A}$ of a twist automorphism of $\int$. Moreover, $b_{\sigma} \varphi=0$; that is, $\varphi$ is a twisted co-cycle. Thus, a triple $\left(\Omega, d, \int\right)$ of the kind we are considering gives rise to a twisted cyclic co-cycle and one can show in the reverse direction that every twisted cyclic co-cycle $\varphi \in C_{\lambda}^{N}(\mathcal{A}, \sigma)$ arises from such a triple $\left(\Omega, d, \int\right)$. 
The construction of differential calculi in the general setting we are considering in this section, and even in the more restricted setting where the algebra $\mathcal{A}$ is the underlying Hopf algebra of a compact quantum group - this is the situation we consider in following sections - is a highly non-trivial problem. The fact that differential calculi correspond to twisted cyclic co-cycles has an extra interest, therefore, since it offers the possibility of constructing calculi by selecting appropriate twisted co-cycles. This sounds promising, but we do not know of any way of obtaining some naturally-occurring differential calculus by this procedure, where the cyclic co-cycle is in some sense given a priori, and one can recognize that this must give rise to an interesting calculus and thereby construct the calculus. However, the idea is worth exploring.

4. Left-invariant linear functionals. In this section we will discuss how twisted graded traces arise naturally in the setting of left-covariant differential calculi over compact quantum groups. These calculi, and the special subclass of bicovariant calculi, are of prime importance in the theory. We begin by defining terms and setting out assumptions.

We specialize our underlying algebra $\mathcal{A}$ now. It will no longer be an arbitrary unital algebra-henceforth, we shall assume it is endowed with a co-multiplication $\Delta$ making it a Hopf algebra. Indeed, we shall later assume that it is the Hopf algebra underlying a compact quantum group and when we talk of a differential calculus over such a quantum group we shall mean a differential calculus over the corresponding Hopf algebra.

A pair $\left(\Omega, \Delta_{L}\right)$ is a left-covariant bi-module over the Hopf algebra $(\mathcal{A}, \Delta)$ if $\Omega$ is a bi-module over $\mathcal{A}$ and $\Delta_{L}$ is a linear map from $\Omega$ to the tensor product $\mathcal{A} \otimes \Omega$ such that the following conditions hold:

(1) $\left(\Delta \otimes \operatorname{id}_{\Omega}\right) \Delta_{L}=\left(\operatorname{id}_{\mathcal{A}} \otimes \Delta_{L}\right) \Delta_{L}$ and $\left(\varepsilon \otimes \operatorname{id}_{\Omega}\right) \Delta_{L}=\operatorname{id}_{\Omega}$, where $\varepsilon$ is the co-unit of $(\mathcal{A}, \Delta)$; that is, $\Delta_{L}$ is a left co-action of $(\mathcal{A}, \Delta)$ on $\Omega$;

(2) $\Delta_{L}(a \omega b)=\Delta(a) \Delta_{L}(\omega) \Delta(b)$, for all $a, b \in \mathcal{A}$ and $\omega \in \Omega$.

An element $\omega \in \Omega$ is left-invariant if $\Delta_{L}(\omega)=1 \otimes \omega$. We denote by $\Omega^{\text {inv }}$ the linear space of all left-invariant elements of $\Omega$ and by $\Omega_{n}^{\text {inv }}$ the linear subspace of all left-invariant elements of dimension $n$.

Now let $(\Omega, d)$ be a differential calculus over $\mathcal{A}$ such that the unit 1 of $\mathcal{A}$ is a unit for $\Omega$ and $d 1=0$. Of course, $\Omega$ is a bi-module over $\mathcal{A}$ in an obvious way. If there is an algebra homomorphism $\Delta_{L}: \Omega \rightarrow \mathcal{A} \otimes \Omega$ making $\Omega$ into a left-covariant bi-module and if $\left(\operatorname{id}_{\mathcal{A}} \otimes d\right) \Delta_{L}=\Delta_{L} d$ and $\Delta_{L}(a)=\Delta(a)$, for all $a \in \mathcal{A}$, we call the triple $\left(\Omega, d, \Delta_{L}\right)$ a left-covariant differential calculus over the Hopf algebra $(\mathcal{A}, \Delta)$. A moment's reflection shows that there can be only one such left co-action $\Delta_{L}$ making $\left(\Omega, d, \Delta_{L}\right)$ a left-covariant differential calculus. For this reason, we will speak of the left-covariant calculus $(\Omega, d)$, omitting explicit reference of the left co-action $\Delta_{L}$. We shall often also speak of the Hopf algebra $\mathcal{A}$, omitting explicit reference of the co-multiplication $\Delta$.

Suppose then $(\Omega, d)$ is a left-covariant differential calculus over a Hopf algebra $\mathcal{A}$. The natural linear functionals to study on $\Omega$ are those that are left-invariant; these are the linear functionals $\int: \Omega \rightarrow \mathbf{C}$ for which 


$$
\left(\operatorname{id}_{\mathcal{A}} \otimes \int\right) \Delta_{L}(\omega)=\left(\int \omega\right) 1,
$$

for all forms $\omega \in \Omega$.

Recall that a (normalized) Haar integral on $\mathcal{A}$ is a linear functional $h: \mathcal{A} \rightarrow \mathbf{C}$ such that $h(1)=1$ and

$$
\left(\operatorname{id}_{\mathcal{A}} \otimes h\right)(\Delta(a))=\left(h \otimes \operatorname{id}_{\mathcal{A}}\right)(\Delta(a))=h(a) 1
$$

for all $a \in \mathcal{A}$. Such a functional does not always exist. However, if $\mathcal{A}$ is the Hopf algebra associated to a compact quantum group, a Haar integral does exist [5, 13]. We shall be interested only in Hopf algebras admitting a Haar integral - these are precisely the co-semisimple Hopf algebras. It is easily seen that at most one Haar integral can exist.

Proposition 4.1. Let $(\Omega, d)$ be a left-covariant differential calculus over a Hopf algebra $\mathcal{A}$ admitting a Haar integral. Let $N$ be a positive integer. There is a non-zero, left-invariant linear functional on $\Omega$ of dimension $N$, unique up to a non-zero scalar factor, if, and only if, the linear space $\Omega_{N}^{\mathrm{inv}}$ is one-dimensional.

We say that a calculus $(\Omega, d)$ is $N$-dimensional if $\Omega_{N} \neq 0$ and $\Omega_{n}=0$, for all $n>N$. The condition that $\Omega_{N}^{\text {inv }}$ be one-dimensional is a natural one for an $N$-dimensional calculus and is often satisfied in examples. The result ensures that in the well-behaved calculi the "volume integrals" that have been defined in a somewhat ad hoc way in the literature are, in fact, the only possible ones, if they are to be left-invariant linear functionals.

Theorem 4.2 (Cf. [6, Corollary 4.9]). Let $(\Omega, d)$ and $\mathcal{A}$ be as in Proposition 4.1. Let $\int: \Omega \rightarrow \mathbf{C}$ be an $N$-dimensional, left-invariant linear functional, for some $N \geq 0$. Suppose also that $\int$ is closed and faithful in the sense that if $\omega$ is a form in $\Omega$ such that $\int \omega^{\prime} \omega=0$, for all $\omega^{\prime} \in \Omega$, then $\omega=0$. Then $\int$ is necessarily a twisted graded trace.

In other terms, any reasonable candidate for a "volume integral" (reasonable in the sense that it is left-invariant, closed and faithful) is necessarily a twisted graded trace - a KMS-type functional.

5. A Hodge-type decomposition and the Dirac and Laplace operators. To introduce the Dirac and Laplace operators we'll need to introduce the co-differential $d^{*}$ of $d$. For this and other reasons we'll need to assume henceforth that the space of leftinvariant forms is finite-dimensional. This is not a strong assumption and is often satisfied in examples. We'll also need to confine ourselves now to differential calculi with a graded involution, since our proofs rely heavily on the existence of this $*$-structure. Again, this is a quite natural assumption in the context of differential calculi over compact quantum groups. Finally, our proofs also require that the underlying Hopf algebra $\mathcal{A}$ also has an involution and admits the existence of a Haar integral. This is, of course, the case for the Hopf algebras associated to compact quantum groups, the case in which we are principally interested. In view of these preliminary remarks, we begin now by defining some terms.

Suppose that $\mathcal{A}$ is a unital $*$-algebra and $(\Omega, d)$ is a differential calculus over $\mathcal{A}$. A graded involution on $\Omega$ is a conjugate-linear map $\omega \mapsto \omega^{*}$ of degree zero such that 
$\left(\omega^{*}\right)^{*}=\omega$, for all $\omega \in \Omega$ and such that

$$
\left(\omega_{k} \omega_{l}\right)^{*}=(-1)^{k l} \omega_{l}^{*} \omega_{k}^{*},
$$

for all $\omega_{k} \in \Omega_{k}$ and $\omega_{l} \in \Omega_{l}$. If $\Omega$ admits a graded involution extending the involution of $\mathcal{A}$ for which $d\left(\omega^{*}\right)=(d \omega)^{*}$, for all $\omega \in \Omega$, then this involution is necessarily unique and we call the pair $(\Omega, d)$ a $*$-differential calculus over the $*$-algebra $\mathcal{A}$.

Henceforth, we assume that $\mathcal{A}$ is a Hopf *-algebra admitting a positive Haar integral $h$ and that $(\Omega, d)$ is a left-covariant $*$-differential calculus over $\mathcal{A}$ such that $\Omega^{\text {inv }}$ is finitedimensional.

That $h$ is positive means $h\left(a^{*} a\right) \geq 0$, for all $a \in \mathcal{A}$. The underlying Hopf $*$-algebra of a compact quantum group always admits a positive Haar integral.

The assumption that $\Omega^{\text {inv }}$ is finite-dimensional ensures that $\Omega$ is $N$-dimensional, for some integer $N$.

We now want to put an inner product structure on $\Omega$. We can do this in a natural way by first recalling that it is a consequence of left-covariance of $\Omega$ that the linear map from $\mathcal{A} \otimes \Omega^{\text {inv }}$ to $\Omega$ that sends $a \otimes \omega$ onto $a \omega$ is a linear isomorphism. We therefore can, and we do, identify these spaces, as linear spaces, using this isomorphism.

Now $\mathcal{A}$ comes naturally endowed with its own inner product defined by setting $(a \mid b)=h\left(b^{*} a\right)$, for all $a, b \in \mathcal{A}$. Since $\Omega^{\text {inv }}$ is finite-dimensional, it too admits inner products. Choose one such that makes the spaces $\Omega_{0}^{\text {inv }}, \Omega_{1}^{\text {inv }}, \ldots, \Omega_{N}^{\text {inv }}$ orthogonal.

Of course, this does not uniquely determine the inner product on $\Omega^{\text {inv }}$. For most of our considerations in this paper, an arbitrary choice such as the one we have made here is all that is needed. However, further restrictions on one's choice are necessary to obtain the strongest results in our theory.

Given our inner products on $\mathcal{A}$ and $\Omega^{\text {inv }}$, we now endow $\Omega$ with the tensor product inner product structure. Thus, we have

$$
(a \omega \mid b \eta)=h\left(b^{*} a\right)(\omega \mid \eta),
$$

for all $a, b \in \mathcal{A}$ and all $\omega, \eta \in \Omega^{\text {inv }}$. We denote by $\omega \mapsto\|\omega\|$ the norm induced by this inner product.

Although generically $\Omega$ is not complete, and the differential operator $d$ is not norm bounded, nevertheless $d$ admits an adjoint $d^{*}$ such that $(d \omega \mid \eta)=\left(\omega \mid d^{*} \eta\right)$, for all $\omega, \eta \in \Omega$. The proof makes use of the fact that $(\Omega, d)$ is a left covariant bi-module. We call $d^{*}$ the co-differential of $d$. We can now define a Dirac operator $D$ and Laplace operator $\nabla$ by setting $D=d+d^{*}$ and $\nabla=D^{2}=d d^{*}+d^{*} d$.

Suppose now $\mathcal{A}$ is the Hopf *-algebra associated to a compact quantum group.

The co-representation theory of compact quantum groups can be used to show two fundamental results concerning these operators. Firstly, $D$ and $\nabla$ are diagonalisable; that is, there is a linear basis for $\Omega$ consisting of eigenvectors of $D$ and $\nabla$; secondly, $\Omega$ admits a Hodge-type decomposition in the sense that

$$
\Omega=\operatorname{ker}(\nabla) \oplus d(\Omega) \oplus d^{*}(\Omega) .
$$

The sum here is orthogonal and the decomposition is an analogue of the classical Hodge decomposition. 
One can also show that

$$
\operatorname{ker}(d)=\operatorname{ker}\left(d^{*} d\right)=\operatorname{ker}(\nabla) \oplus d(\Omega) \text { and } \operatorname{ker}\left(d^{*}\right)=\operatorname{ker}\left(d d^{*}\right)=\operatorname{ker}(\nabla) \oplus d^{*}(\Omega) .
$$

The elements of $\operatorname{ker}(\nabla)$ are called the harmonic forms. By the Hodge decomposition, if $\omega \in \Omega$, then $\omega=\omega_{0}+d \omega_{1}+d^{*} \omega_{2}$, for some harmonic form $\omega_{0} \in \operatorname{ker}(\nabla)$ and some forms $\omega_{1}, \omega_{2} \in \Omega$. We call $\omega_{0}$ the harmonic part of $\omega$. Note that we also show in [7] that $\operatorname{ker}(\nabla)=\operatorname{ker}(d) \cap \operatorname{ker}\left(d^{*}\right)$. It follows that $d \omega=0$ if, and only if, $\omega=\omega_{0}+d \omega_{1}$.

Our Hodge-type decomposition allows one to deduce a version of a classical theorem of de Rham identifying the de Rham cohomology space of dimension $k$ with the corresponding space of harmonic forms of dimension $k$. To see this, denote by $H^{*}(\Omega)$ the cohomology associated to the de Rham complex

$$
0 \rightarrow \Omega_{0} \stackrel{d}{\rightarrow} \Omega_{1} \stackrel{d}{\rightarrow} \cdots \stackrel{d}{\rightarrow} \Omega_{N} \rightarrow 0
$$

Let $[\omega]$ denote the class of the form $\omega \in \Omega_{k}$ in $H^{k}(\Omega)$. Then the natural map, $[\omega] \mapsto \omega_{0}$, where $\omega_{0}$ is the harmonic part of $\omega$, is a well-defined linear isomorphism from $H^{k}(\Omega)$ onto the space of harmonic $k$-forms $\operatorname{ker}\left(\nabla_{k}\right)$. Here $\nabla_{k}$ is the restriction of $\nabla$ to $\Omega_{k}$. The proof is quite straightforward, but since the result is not included in our papers $[6,7]$ we give the details here: First, to see the map is well defined, suppose that $\omega$ and $\eta$ are $k$-forms for which $[\omega]=[\eta]$. Then $\omega-\eta$ is exact; that is, $\omega-\eta=d \theta$, for some form $\theta$. Since the harmonic part of $\omega-\eta$ is clearly $\omega_{0}-\eta_{0}$, where $\omega_{0}$ and $\eta_{0}$ are the harmonic parts of $\omega$ and $\eta$, respectively, we must have $\omega_{0}-\eta_{0}=0$, by uniqueness of the Hodge decomposition of $\omega-\eta$. Thus, $\omega_{0}=\eta_{0}$ and our map from $H^{k}(\Omega)$ to $\operatorname{ker}\left(\nabla_{k}\right)$ is well defined. Its linearity and surjectivity are obvious. To see injectivity, let $\omega$ be a form in $\Omega_{k}$ such that $d \omega=0$ and its harmonic part $\omega_{0}$ vanishes. Then $\omega=d \omega_{1}+d^{*} \omega_{2}$, for some forms $\omega_{1}, \omega_{2} \in \Omega$. The equation $d \omega=0$ implies $d d^{*} \omega_{2}=0$. Hence, $d^{*} \omega_{2}=0$. Therefore, $\omega=d \omega_{1}$ and so $[\omega]=0$, as required.

If $H^{*}(\Omega)$ is finite-dimensional, we can now define an Euler-Poincaré characteristic $\chi(\Omega)$ for $\Omega$ using the usual formula; that is, by setting

$$
\chi(\Omega)=\sum_{k=0}^{N}(-1)^{k} \operatorname{dim}\left(H^{k}(\Omega)\right) .
$$

Likewise, we can define the analytic index ind $(\nabla)$ of $\nabla$ using the classical formula; that is, by setting

$$
\text { ind }(\nabla)=\sum_{k=0}^{N}(-1)^{k} \operatorname{dim}\left(\operatorname{ker}\left(\nabla_{k}\right)\right) \text {. }
$$

Our de Rham theorem then gives a simple analogue of the Atiyah-Singer index theorem in this setting, identifying a geometric index of the quantum differential structure with an operator index of the Laplacian: $\chi(\Omega)=$ ind $(\nabla)$.

For other results on a Hodge-type decomposition and on de Rham cohomology in the setting of differential calculi over quantum groups, see [3].

6. The Hodge operator and Poincaré duality. In this section we discuss the Hodge operator and a version of Poincaré duality. First we need to make some additional assumptions. 
We shall suppose that the following conditions hold (in addition to our standing assumptions from the preceding section-we include the assumption that $\mathcal{A}$ is the Hopf *-algebra associated to a compact quantum group):

(1) $(\Omega, d)$ is non-degenerate in the sense that if $\omega \in \Omega_{k}$ and $\omega^{\prime} \omega=0$, for all $\omega^{\prime} \in \Omega_{N-k}$, then $\omega=0$;

(2) $\operatorname{dim}\left(\Omega_{N}^{\text {inv }}\right)=1$ and $d\left(\Omega_{N-1}^{\text {inv }}\right)=0$.

These conditions imply that $(\Omega, d)$ admits an $N$-dimensional, faithful, left-invariant, closed, twisted graded trace $\int$, unique up to multiplication by a non-zero scalar. (In fact, Conditions (1) and (2) are equivalent to assuming that $(\Omega, d)$ admits an $N$-dimensional, faithful, closed, left-invariant linear functional.)

The conditions imply the existence of an extremely useful operator: There is a unique left $\mathcal{A}$-linear isomorphism $L: \Omega \rightarrow \Omega$, called the Hodge operator, such that $L\left(\Omega_{k}\right)=$ $\Omega_{N-k}$, for $k=0, \ldots, N$ and

$$
(\omega \mid \eta)=\int \eta^{*} \wedge L(\omega)
$$

for all $\omega, \eta \in \Omega$. Moreover, if $\omega \in \Omega_{k}$, then $d^{*}(\omega)=(-1)^{k} L^{-1} d L(\omega)$.

The results of the preceding two paragraphs follow from [6] and [7]. It is also possible to obtain a version of Poincaré duality; since this is a new result, we state it formally here and give its proof.

Theorem 6.1 (Poincaré Duality). The map

$$
H^{k}(\Omega) \times H^{N-k}(\Omega) \rightarrow \mathbf{C}, \quad([\omega],[\eta]) \mapsto \int \omega \wedge \eta,
$$

is well-defined, bilinear and non-degenerate.

Proof. To see the map is well defined, suppose that $[\eta]=\left[\eta^{\prime}\right]$, so that $\eta-\eta^{\prime}=d \theta$, for some form $\theta$. Then

$$
\int \omega \wedge \eta-\int \omega \wedge \eta^{\prime}=\int \omega \wedge d \theta
$$

We need to show the right-hand side integral is equal to zero. This follows from the fact that $d \omega=0$, that $\int d=0$ and the equation

$$
d(\omega \wedge \theta)=d \omega \wedge \theta+(-1)^{k} \omega \wedge d \theta .
$$

A similar proof shows that if $[\omega]=\left[\omega^{\prime}\right]$, then $\int \omega \wedge \eta=\int \omega^{\prime} \wedge \eta$. Bilinearity of $([\omega],[\eta]) \mapsto$ $\int \omega \wedge \eta$ is, of course, obvious.

Suppose now $[\omega] \in H^{k}(\omega)$ and $\int \omega \wedge \eta=0$, for all $[\eta] \in H^{N-k}(\Omega)$. Then, if $\theta \in$ $\operatorname{ker}\left(d^{*}\right) \cap \Omega_{k}$, we have $d L(\theta)=(-1)^{k} L d^{*} \theta=0$ and therefore $\left(\theta \mid \omega^{*}\right)=\int \omega \wedge L(\theta)=0$. Consequently, since $\omega^{*} \in \Omega_{k}$ and the spaces $\Omega_{0}, \ldots, \Omega_{N}$ are orthogonal, $\omega^{*}$ is orthogonal to $\operatorname{ker}\left(d^{*}\right)=\operatorname{ker}(\nabla) \oplus d^{*}(\Omega)$ and therefore $\omega^{*}=d \omega^{\prime}$, for some element $\omega^{\prime} \in \Omega$, by our Hodge-type decomposition. Hence, $\omega \in d(\Omega)$ and therefore $[\omega]=0$. A similar proof shows that if $[\eta] \in H^{N-k}(\Omega)$ and $\int \omega \wedge \eta=0$, for all $[\omega] \in H^{k}(\Omega)$, then $[\eta]=0$. Thus, the map $([\omega],[\eta]) \mapsto \int \omega \wedge \eta$ is non-degenerate, as required.

If the cohomology spaces $H^{k}(\Omega)$ are finite-dimensional, the preceding theorem implies that $H^{k}(\Omega)$ is linearly isomorphic to $H^{N-k}(\Omega)$, for $k=0, \ldots, N$. However, this is still 
true even in the case that the cohomology spaces are not finite-dimensional. To see this we observe first that by replacing, if necessary, the inner product on $\Omega^{\text {inv }}$ by another one (for which the spaces $\Omega_{1}^{\text {inv }}, \ldots, \Omega_{N}^{\text {inv }}$ are still pairwise orthogonal), we may obtain the equality

$$
d \omega=(-1)^{k+1} L^{-1} d^{*} L \omega \quad\left(\omega \in \Omega_{k}\right),
$$

a result that we shall need in the following proof (for this result see [7]). We now explicitly define a map $\psi: H^{k}(\Omega) \rightarrow H^{N-k}(\Omega)$ by setting $\psi[\omega]=\left[L \omega_{0}\right]$, where $\omega_{0}$ is the harmonic part of $\omega$. It is clear that $\psi$ is well defined and linear. To see that it is injective, suppose that $\psi[\omega]=0$; that is, $\left[L \omega_{0}\right]=0$. Then $L \omega_{0}=d \theta$, for some form $\theta \in \Omega_{N-k-1}$. Hence, $\omega_{0}=L^{-1} d \theta=L^{-1} d L L^{-1} \theta=(-1)^{k+1} d^{*} L^{-1} \theta$. Since $\operatorname{ker}(\nabla)$ and $d^{*}(\Omega)$ are orthogonal, this shows that $\omega_{0}=0$ and therefore, since $d \omega=0$, we have $\omega \in d(\Omega)$. Hence, $[\omega]=0$ and $\psi$ is injective, as required. To see surjectivity of $\psi$, let $[\eta]$ be an element of $H^{N-k}(\Omega)$. Without loss of generality, we may suppose that $\eta$ is harmonic, so that $d \eta=d^{*} \eta=0$. Choose $\omega \in \Omega_{k}$ such that $L(\omega)=\eta$. Since $d \omega=(-1)^{k+1} L^{-1} d^{*} L \omega=(-1)^{k+1} L^{-1} d^{*} \eta=0$, we have $[\omega] \in H^{k}(\Omega)$. Since we also have $d^{*} \omega=(-1)^{k} L^{-1} d L \omega=(-1)^{k} L^{-1} d \eta=0, \omega$ is a harmonic form. Hence, $\psi[\omega]=[L(\omega)]=[\eta]$. Thus, $\psi$ is surjective.

Note that we needed to make a choice of a particular inner product on $\Omega^{\text {inv }}$ so that Equation (6) holds in order to make the proof above work, and that this choice affects how $L$ is defined, and therefore how $\psi$ is defined. Nevertheless, this choice does not enter into the statement of our result if we restate it less precisely, to say only that $H^{k}(\Omega)$ and $H^{N-k}(\Omega)$ are linearly isomorphic, for $k=0, \ldots, N$.

Henceforth, we return to our general assumption concerning the inner product on $\Omega^{\text {inv }}$; that is, we assume only that it is such that the the spaces $\Omega_{1}^{\text {inv }}, \ldots, \Omega_{N}^{\text {inv }}$ are pairwise orthogonal.

It follows from our Poincaré duality theorem that if the calculus $(\Omega, d)$ has finite Euler-Poincaré characteristic - that is, the spaces $H^{k}(\Omega)$ are finite-dimensional - and $N$ is odd, then $\Omega$ has zero characteristic; that is, $\chi(\Omega)=0$.

For other results on the Hodge and Laplace operators in the setting of differential calculi over quantum groups, see [2].

7. $K$-cycles. Recall that Connes $[1$, p. 310$]$ defines a $K$-cycle over a $*$-algebra $A$ to be a triple $(H, D, \pi)$ for which

(1) $H$ is a Hilbert space and $\pi: A \rightarrow B(H)$ is a $*$-homomorphism;

(2) $D$ is an (unbounded) self-adjoint operator in $H$ with compact resolvent;

(3) $[D, \pi(a)]$ is norm-bounded, for all $a \in A$.

Continuing with the notation and assumptions from the preceding two sections, let $H$ be the Hilbert space completion of $\Omega$. Define a $*$-homomorphism $\pi: \mathcal{A} \rightarrow B(H)$ by setting $\pi(a) \omega=a \omega$, for all $a \in \mathcal{A}$ and $\omega \in \Omega$. If $D$, as before, denotes our Dirac operator, then one might expect (hope) the triple $(H, D, \pi)$ is a $K$-cycle. (More precisely, one should replace $D$ by an extension that is self-adjoint in $H$, but we'll ignore all finer points here.) However, in view of our observations in earlier sections, one should not now be surprised to find that $(H, D, \pi)$ is not, in general, a $K$-cycle. The principal difficulty involves Condition (3) in Connes's definition - this does not hold in general. We shall 
sketch a proof in one special case, since the details are rather simple there. For a fuller discussion of "no-go" results along these lines we refer the reader to the papers $[9,10]$ of K. Schmüdgen.

Let $(\Omega, d)$ be the left-covariant calculus over the quantum group $S U_{q}(2)$ constructed by Woronowicz in [11]. Let $\mathcal{A}_{q}$ be the Hopf *-algebra underlying $S U_{q}(2)$. The assumptions of the preceding two sections are satisfied by $(\Omega, d)$ and $\mathcal{A}_{q}$. However, $[D, \pi(a)]$ is not normbounded for every element $a \in \mathcal{A}_{q}$. Suppose otherwise and we shall obtain a contradiction. In this case the map $\psi_{a}: \Omega_{0} \rightarrow \Omega_{1}$, defined by restricting $[D, \pi(a)]$, is norm-bounded. If $b \in \Omega_{0}$, then $\psi_{a}(b)=d(a b)-a d b=d(a) b$, using the fact that $d^{*}\left(\Omega_{0}\right)=0$. Since the elements of the form $a d a^{\prime}\left(a, a^{\prime} \in \mathcal{A}_{q}\right)$ linearly span $\Omega_{1}$, we have norm-boundedness of the map, $\Omega_{0} \rightarrow \Omega_{1}, b \mapsto \omega b$, for all $\omega \in \Omega_{1}$. Now $\Omega_{1}^{\text {inv }}$ admits a linear basis $\omega_{0}, \omega_{1}, \omega_{2}$ and there exist unique linear maps $E_{r}: \Omega_{0} \rightarrow \Omega_{0}$ such that $\omega_{r} b=E_{r}(b) \omega_{r}$, for $r=0,1,2$. Normboundedness of the maps $b \mapsto \omega_{r} b$ implies norm-boundedness of the maps $b \mapsto E_{r}(b) \omega_{r}$ which in turn implies norm-boundedness of the maps $E_{r}$. However, it is easy to verify, using the formulas in Table 1 of [11], that the maps $E_{r}$ are not norm-bounded. Hence, $[D, \pi(a)]$ cannot be norm-bounded for all $a \in \mathcal{A}_{q}$.

The preceding counter-example raises the question of what is possible. To address this question we were led to the problem of computing the eigenvalues of the Laplacian. I'll explain the connection to $K$-cycles below, but first I'll discuss briefly this problem. It is an interesting one and one that is quite difficult in general. However, there is one case where the computation of the eigenvalues is not so difficult, at least in principle. This concerns the problem of computing the eigenvalues of $\nabla_{0}$, the restriction of $\nabla$ to the 0-forms.

Suppose then $(\Omega, d)$ is a left-covariant $*$-differential calculus over a Hopf $*$-algebra $\mathcal{A}$ and, for simplicity, assume that $(\Omega, d)$ and $\mathcal{A}$ satisfy the assumptions of Sections 5 and 6 . In this case there exists an orthonormal basis $\omega_{1}, \ldots, \omega_{M}$ for $\Omega_{1}^{\text {inv }}$ and linear functionals $\chi_{1}, \ldots, \chi_{M}$ on $\mathcal{A}$ such that

$$
d a=\sum_{r=1}^{M}\left(\left(\operatorname{id}_{\mathcal{A}} \otimes \chi_{r}\right) \Delta(a)\right) \omega_{r} \quad(a \in \mathcal{A}) .
$$

Set $\chi=\sum_{r=1}^{M}\left(\chi_{r}^{*} \otimes \chi_{r}\right) \Delta$, where $\chi_{r}^{*}(a)=\chi_{r}\left((S(a))^{*}\right)^{-}$, for all $a \in \mathcal{A}(S$ is the antipode of $\mathcal{A})$. Then it is straightforward to calculate that $\nabla_{0}(a)=\left(\operatorname{id}_{\mathcal{A}} \otimes \chi\right) \Delta(a)$, for all $a \in \mathcal{A}$.

Suppose now that $U$ is an irreducible unitary co-representation of $\mathcal{A}$ on the finitedimensional Hilbert space $\mathbf{C}^{n}$. Then the operator $T=(\mathrm{id} \otimes \chi)(U) \in B\left(\mathbf{C}^{n}\right)$ is positive. Choose an orthonormal basis $e_{1}, \ldots, e_{n}$ for $\mathbf{C}^{n}$ for which $T$ has diagonal matrix, so that $T e_{i}=\lambda_{i} e_{i}$, for some scalars $\lambda_{i}$. Let $U_{i j}$ be the matrix entries of $U$ relative to this basis. Then the matrix $\left(T_{i j}\right)$ of $T$ is given by $T_{i j}=\chi\left(U_{i j}\right)=\lambda_{i} \delta_{i j}$. Hence, $\nabla\left(U_{i j}\right)=\left(\operatorname{id}_{\mathcal{A}} \otimes\right.$ $\chi) \Delta\left(U_{i j}\right)=\left(\operatorname{id}_{\mathcal{A}} \otimes \chi\right)\left(\sum_{k=1}^{n} U_{i k} \otimes U_{k j}\right)=\sum_{k=1}^{n} \chi\left(U_{k j}\right) U_{i k}=\sum_{k=1}^{n} \lambda_{k} \delta_{k j} U_{i k}=\lambda_{j} U_{i j}$. Thus, $U_{i j}$ is an eigenvector of $\nabla_{0}$ with eigenvalue $\lambda_{j}$.

Since $\mathcal{A}$ admits a linear basis consisting of the matrix entries of a family of pairwiseinequivalent irreducible unitary co-representations of $\mathcal{A}$, it follows that $\nabla_{0}$ is diagonalisable. Moreover, we can calculate the eigenvalues provided we can calculate explicitly $\chi$ and a complete family of inequivalent irreducible co-representations. 
This is the easy bit - calculating the eigenvalues of $\nabla$ on $\Omega_{0}$. Things become much, much more complicated when one tries to calculate the eigenvalues of $\nabla$ on the spaces $\Omega_{1}, \Omega_{2}, \ldots$ However, we were able to do this in the case of Woronowicz' first example of a three-dimensional differential calculus $(\Omega, d)$ over the Hopf $*$-algebra $\mathcal{A}_{q}$ underlying the compact quantum group $S U_{q}(2)$. This is the calculus already mentioned above, introduced in [11]. We know already that in this case $[D, \pi(a)]$ is not norm-bounded for all $a \in \mathcal{A}_{q}$. However, we succeeded in showing that if $\alpha$ and $\beta$ are non-negative numbers such that $\alpha+\beta=1$, and if $R$ is the norm-bounded operator on $\Omega$ defined by $R=(1+\nabla)^{-1 / 2}$, then $R^{\alpha}[D, \pi(a)] R^{\beta}$ is norm-bounded for all $a \in \mathcal{A}_{q}$. Here, as before, $\pi$ is the $*$-homomorphism from $\mathcal{A}_{q}$ to $B(H)$ defined by left multiplication on $\Omega$ and $H$ is the Hilbert space completion of $\Omega$. This boundedness result required our calculation of the eigenvalues of $\nabla$ and the derivation of asymptotic estimates on these eigenvalues. The proofs are lengthy and quite complicated.

Our boundedness result is interesting for a number of reasons. Firstly, A. Jaffe has developed in [4] an extension of Connes' theory to cover the case where $[D, \pi(a)]$ may not be norm-bounded, but where, for some positive norm-bounded operator $R$ and some non-negative numbers $\alpha$ and $\beta, R^{\alpha}[D, \pi(a)] R^{\beta}$ is norm-bounded, for all $a$ in the relevant algebra. Unfortunately, Jaffe requires that $\alpha+\beta<1$ ! Therefore, his theory does not apply to this example, no more than Connes' does. However, it is possible that the asymptotic estimates we derived could be refined to show that we could take $\alpha+\beta<1$ and thereby fit within Jaffe's framework (personally I doubt this). An alternative possibility is to try to extend Jaffe's theory to cover the case where $\alpha+\beta=1$. There is probably a very good reason why Jaffe did not do this in the first place and I suspect this to be a very hard problem, if it is do-able at all.

To conclude, our results show the limitations of existing theories of noncommutative geometry in the context of differential calculi over compact quantum groups. Nevertheless, many results of the classical theory of manifolds - the Hodge decomposition, de Rham's theorem, Poincaré duality - can be extended to our setting, as can modified versions of some aspects of Connes' theory - twisted graded traces and twisted cyclic cohomology. Moreover, our calculations offer some possible directions for how Connes' and Jaffe's theories might be modified to cover the case of differential calculi over quantum groups.

\section{References}

[1] A. Connes, Noncommutative Geometry, Academic Press, San Diego, 1994.

[2] I. Heckenberger, Hodge and Laplace-Beltrami operators for bicovariant differential calculi on quantum groups, Compositio Math. 123 (2000), 329-354.

[3] I. Heckenberger and A. Schüler, De Rham cohomology and Hodge decomposition for quantum groups, preprint, University of Leipzig, 2000.

[4] A. Jaffe, Quantum harmonic analysis and geometric invariants, Advances in Math. 143 (1999), 1-110.

[5] A. Klimyk and K. Schmüdgen, Quantum Groups and their Representations, Springer, Heidelberg-New York, 1997. 
[6] J. Kustermans, G. J. Murphy and L. Tuset, Differential calculi over quantum groups and twisted cyclic cocycles, J. Geom. Phys. 44 (2003), 570-594.

[7] J. Kustermans, G. J. Murphy and L. Tuset, Quantum groups, differential calculi and the eigenvalues of the Laplacian, preprint, National University of Ireland, Cork, 2000. arXiv:math.QA/0111042.

[8] G. J. Murphy and L. Tuset, Compact quantum groups, preprint, National University of Ireland, Cork, 1999.

[9] K. Schmüdgen, Commutator representations of differential calculi on the quantum group $S U_{q}(2)$, J. Geom. Phys., 31 (1999), 241-264.

[10] K. Schmüdgen, Commutator representations of covariant differential calculus on quantum groups, Lett. Math. Phys. 59 (2002), 95-106.

[11] S. L. Woronowicz, Twisted SU(2) group - an example of a noncommutative differential calculus, Publ. RIMS Kyoto Univ. 23 (1987), 117-181.

[12] S. L. Woronowicz, Differential calculus on compact matrix pseudogroups (quantum groups), Commun. Math. Phys. 122 (1989), 125-170.

[13] S. L. Woronowicz, Compact quantum groups, in: Symétries Quantiques, North-Holland, Amsterdam, 1998, 845-884. 